https://doi.org/10.15407/ujpe63.8.740

O. NOVAK, ${ }^{1}$ R. KHOLODOV, ${ }^{1}$ A. FOMINA ${ }^{2}$

${ }^{1}$ Institute of Applied Physics, Nat. Acad. of Sci. of Ukraine

(58, Petropavlivs'ka Str., Sumy 40030,Ukraine; e-mail: novak-o-p@ukr.net)

2 Bogolyubov Institute for Theoretical Physics, Nat. Acad. of Sci. of Ukraine

(14b, Metrolohichna Str., Kyiv 03143, Ukraine; e-mail: afomina@bitp.kiev.ua)

\title{
ROLE OF DOUBLE LAYERS IN THE FORMATION OF CONDITIONS FOR A POLARIZATION PHASE TRANSITION TO THE SUPERRADIANCE STATE IN THE IO FLUX TUBE
}

\begin{abstract}
A possibility of the electron phase transition into cyclotron superradiance mode in a vicinity of the Io flux tube foot in the Jovian magnetosphere has been considered. A high power of cyclotron superradiance allows it to be considered as the main mechanism of decameter Jupiter radiation generation in the form of S-bursts. It was found that the downward electron beams emitted by Io are able to create electric double layers in the form of shock waves. Such waves, when moving along the flux tube, accelerate electrons in the magnetosphere. As a result, the temperature of the electron plasma component decreases considerably. The emerging upward electron beams create conditions favorable for the phase transition into the cyclotron superradiance mode to take place.
\end{abstract}

Ke ywords: magnetosphere, superradiance, double layer, plasma.

\section{Introduction}

Jupiter is a powerful source of the non-thermal radiation emission in the solar system with intense bursts in the decameter wavelength interval. Short bursts of decameter radiation (S-bursts) attract attention of scientists because of such interesting characteristics as a high brightness temperature that reaches $T_{b} \sim 10^{7} \mathrm{~K}$, a quasiperiodic structure, and a correlation between the probability of the burst emergence and the position of Io, the Jovian satellite $[1,2]$.

According to observation data, the source of Sbursts is located near the foot of the Io magnetic flux tube in the Jovian ionosphere [1,3]. A negative frequency drift of the bursts indicates that the source activity is associated with electron fluxes that move upward from Jupiter into a region with a weaker lo-

(C) O. NOVAK, R. KHOLODOV, A. FOMINA, 2018 cal magnetic field. At present, there is no generally accepted model of S-burst source.

According to the existing concepts concerning the Jovian magnetosphere, the fluxes of high-energy electrons are formed as a result of the acceleration by the induced electromagnetic force of the Jovian satellite Io (of about $400 \mathrm{keV}$ ) and move toward Jupiter along the force lines of the magnetic field, forming the Io flux tube. These fluxes of charged particles are accelerated between the satellite and the planet, and generate aurorae near the magnetic poles of the planet. The Io tube foot looks like a bright spot in the ultraviolet spectral interval on the Jupiter surface [4]. Note that NASA's Juno spacecraft reached the Jovian orbit in 2016. This apparatus has tools for measuring the direction and energy of charged particle fluxes. The data obtained from Juno showed that the character of electron fluxes over the Jovian

ISSN 2071-0194. Ukr. J. Phys. 2018. Vol. 63, No. 8 
poles differs significantly from those predicted in the framework of available models of the Jovian magnetosphere. In particular, upward electron fluxes were also discovered over the polar caps [5].

In works [6-10], a model was proposed that describes the generation of a powerful decameter radiation in the Jupiter-Io system. It is based on the effect of the Fomin-Dicke collective coherent cyclotron superradiance. The superradiance phenomenon was predicted for the first time for solids in work [11]. In works [6,7], its possibility was substantiated for a system of inverted electrons at high Landau levels in rarefied magnetized plasma. In this case, the electron energy is quantized,

$E_{\perp} \approx l \hbar \omega_{H}$,

where $\omega_{H}=e H /(m c)$ is the cyclotron frequency, and $l \gg 1$ is the ordinal number of the Landau level. It was shown that there is a polarization phase transition into the cyclotron superradiance (CSR) state within "coherence domains" whose dimensions are much smaller than the cyclotron radiation wavelength, $R_{0} \ll \lambda_{H}$, and whose concentration of inverted electrons exceeds a certain threshold value $n_{e c}$. Namely, due to the dipole-dipole interaction, the dipole moments of electrons, which rotate at the frequency $\omega_{H}$ in a domain, become cophased with respect to their directions. As a result, the intensity of their collective coherent radiance becomes proportional to the square of their number.

From an equation of the Weiss type for the determination of the spontaneous domain polarization, a criterion of the phase transition into the CSR mode was found:

$\chi=\frac{m c^{2}}{H^{2}} \frac{n \bar{E}_{\perp}}{k T}$,

where $n$ is the electron concentration, $T$ the electron temperature, and $\bar{E}_{\perp}$ a characteristic transverse energy of electrons. The SR mode is possible, if $\chi \gg 1$.

The essence of the model proposed for the source of S-bursts is as follows. The accelerating potential of Io creates fluxes of high-energy electrons, which move along the Io flux tube toward Jupiter. When those fluxes interact with planet's ionosphere, there arise upward electron beams, probably as a result of the formation of double electric layers in the magnetized plasma of the Jovian magnetosphere. In their turn, the electron bunches, when rising upward along the Io tube at a rate that determines the negative drift magnitude of the decameter S-spectra, transit into the CSR state and emit coherently.

The parameters of upward beams, in particular, their temperature and concentration, are crucial for the model of S-burst source, because they determine a possibility of the phase transition into the CSR mode. At present, the precise position of the active region, where the decameter radiation bursts are generated, and the conditions in this region are unknown. Assuming the temperature $T \approx 10^{3} \mathrm{~K}$ and the transverse energy $\bar{E}_{\perp} \approx 1 \mathrm{keV}$ [8], we obtain that the critical electron concentration, above which the CSR will occur, is equal to about $2 \times 10^{3} \mathrm{~cm}^{-3}$. Although such values seem plausible for the Jovian magnetosphere, a sufficient excess of the electron concentration over the critical value is required for the SR mode to be effective. Hence, the search for mechanisms that could provide a better quality of upward beams satisfying criterion (2) remains to be a challenging task.

In this paper, we considered processes that could improve conditions for the realization of the CSR effect near Jupiter. In Section 2, the influence of the acceleration undergone by upward electron beams in the plasma double layers on the realization conditions of the CSR effect is considered. In Section 3, a possibility of the formation of double layers with required parameters as a result of the interaction between downward electron fluxes in the Io tube and the plasma of the Jovian ionosphere is discussed.

\section{Influence of the Acceleration on the CSR Criterion}

Let us consider the processes that can facilitate the electron transition into the CSR mode in the burst source region. For simplicity, we assume that the magnetic field completely suppresses the transverse motion. As a result, a simple one-dimensional model can be used to describe the phenomena in plasma in the flux tube.

The so-called electric double layers [14-21] are known to play an important role in the processes running in the magnetospheres of planets. The double electric layer is a plasma region with a violated quasineutrality. The spatial charge separation in this region results in the appearance of a local 


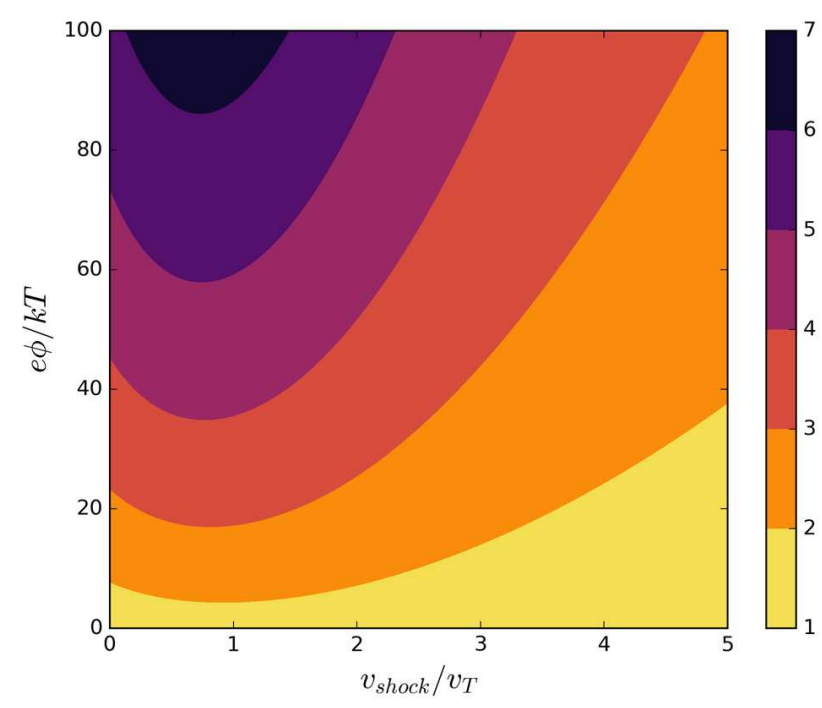

Fig. 1. Variation of the parameter for the transition into the CSR mode under the action of the electrostatic acceleration

electric field. The mechanisms governing the formation of double layers are quite diverse [15]. In particular, ion-acoustic and shock waves in plasma can create a finite potential difference. In work [14], it was shown that the Vlasov and Poisson equations have a purely electrostatic solution, which looks like a shock wave with a monotonically increasing potential, and it is stationary in the comoving reference frame.

The electric field in the double layer can accelerate electrons in the magnetospheric plasma. Due to the electrostatic acceleration, the effective temperature of an accelerated beam is reduced. This phenomenon is well known in the physics of accelerators, in particular, in the electron cooling problems $[12,13]$. Note that the resulting velocity distribution differs from the Maxwellian one. Nevertheless, we may talk about the effective temperature $T$ that is defined as the distribution dispersion: $k T=m\left(\overline{v^{2}}-\bar{v}^{2}\right) / 2$.

Since the criterion of the CSR mode realization is inversely proportional to the temperature, the cooling of the electron plasma component favors the transition into the superradiance mode. Really, superradiance emerges owing to the cophasing of electrons rotating around the force lines of the magnetic field. Thermally induced chaotic collisions evidently hinder this process. Therefore, the transition into the CSR mode is possible only if the temperature is low enough.
On the other hand, according to the continuity equation, the concentration of accelerated electrons decreases, which interferes with the CSR. But the competing cooling and concentration reduction effects depend differently on the accelerating potential $\phi_{0}$. Hence, generally speaking, the electrostatic acceleration in the double layer may be beneficial for the CSR, depending on which of the processes dominates.

For a more detailed analysis, we calculated how the quantity $n / k T$ characterizing the electron component of the magnetospheric plasma depends on the shock wave parameters: the shock wave velocity and the accelerating potential in the double layer.

Figure 1 exhibits a contour plot of the ratio $\chi / \chi_{0}$ in the $\left(\phi_{0}, v\right)$-plane. For a fixed velocity value, the $\chi / \chi_{0}$-magnitude monotonically grows together with $\phi_{0}$. One can see that the effect is maximum at a drift velocity (the shock wave propagation) that is approximately equal to the thermal velocity of plasma electrons, $v \approx v_{T}$. Note that $v_{T}$ is a characteristic velocity of electrostatic waves in plasma. Hence, the effect is the most pronounced in the characteristic interval of wave propagation velocities in plasma. The effect intensity depends on the characteristic values of the potential in the double layers. The analysis of their properties is carried out in the next section.

\section{Mechanism of Double Layer Formation}

Although the precise position of the source of decameter radiation bursts is unknown, it is generally accepted that the source is located in the region of the Io flux tube foot. It is natural to assume that the required shock waves are formed as a result of the interaction between the Jovian magnetosphere and electron beams accelerated by the Io potential along the flux tube in the direction to the Jovian poles. In particular, in work [14], it was shown that the Vlasov and Poisson equations admit a purely electrostatic formation mechanism of a shock wave with a monotonic potential.

Let us apply the method of work [14] to analyze possible accelerating potential values. For this purpose, we should change to the reference system comoving with the shock wave. In this system, the picture is stationary. Let us consider the laminar mechanism of double layer formation with a potential that monotonically increases from $\phi=0$ at $x=-\infty$ to a certain value $\phi_{0}$ at $x=+\infty$. For simplicity, we

ISSN 2071-0194. Ukr. J. Phys. 2018. Vol. 63, No. 8 
consider only the electron component. The ions are assumed to be uniformly distributed in the space,

$n_{i}=n_{0}=$ const.

We also assume that the distribution function of the electron component depends only on the total energy

$E=\frac{m v^{2}}{2}-e \phi=$ const

where $e$ is the elementary charge.

Let us introduce two components of the electron distribution according to the sign of the total energy $E$. Electrons with positive energies can overcome the potential barrier of the double layer; they constitute the free component. Electrons belonging to the trapped component have a negative total energy, and they can only be to the right from the potential change (Fig. 2, lower panel).

It is natural to identify the free component with magnetospheric electrons and put their distribution function at $x=-\infty$ to be equal to the equilibrium one. Hence, the free component concentration equals

$n_{f}(x, v) \stackrel{x \rightarrow-\infty}{\longrightarrow} \frac{n_{0}}{v_{T} \sqrt{2 \pi}} \exp \left[-\frac{\left(v-v_{d}\right)^{2}}{2 v_{T}^{2}}\right]$,

where the drift velocity $v_{d}$ corresponds to the shock wave velocity along the Io flux tube. By expressing the velocity $v$ in terms of the energy $E$, we can find the electron distribution at an arbitrary point $x$ :

$n_{f}(x, v)=\frac{n_{0}}{v_{T} \sqrt{2 \pi}} e^{-\frac{1}{2 v_{T}^{2}}\left(\sqrt{v^{2}-2 \frac{e \phi}{m}}+\sigma v_{d}\right)^{2}}$.

Here, the notation $\sigma=\operatorname{sign}(v)= \pm 1$ is used, and the velocity $v$ satisfies the condition $|v|>v_{\phi}$, where

$v_{\phi}=\sqrt{\frac{2 e \phi}{m}}$.

The concentration at an arbitrary point $x$ can be easily calculated by integrating Eq. (6) over the velocity $v$. Changing to the dimensionless velocity $u=v / v_{T}$, we obtain

$n_{f}(x)=\frac{n_{0}}{\sqrt{2 \pi}} \sum_{\sigma= \pm 1} \int_{u_{\phi}}^{\infty} e^{-\frac{1}{2}\left(\sqrt{u^{2}-u_{\phi}^{2}}-\sigma u_{d}\right)^{2}} d u$.

Note that the concentration $n_{f}(x)$ is an implicit function of the coordinate, so that it can be considered as

ISSN 2071-0194. Ukr. J. Phys. 2018. Vol. 63, No. 8 a function of the potential $\phi(x)$. For the convenience of further calculations, let us change to the integration over the interval $[0, \infty)$ by changing to the variable $t=u-u_{\phi}$. Then

$n_{f}(\Psi)=\frac{n_{0}}{\sqrt{2 \pi}} \sum_{\sigma= \pm 1} \int_{0}^{\infty} e^{-\frac{1}{2}\left(\sqrt{t^{2}+t \sqrt{8 \Psi}}-\sigma u_{d}\right)^{2}} d t$

where the dimensionless variables

$\Psi=\frac{e \phi}{k T}, \quad \xi=\frac{x}{\lambda_{\mathrm{D}}}$

are introduced, and $\lambda_{\mathrm{D}}$ is the Debye radius.

The trapped component concentration as a function of $\phi(x)$ can be expressed in the form

$n_{e f}(\phi)=2 \int_{-e \phi}^{0} \frac{n_{t}(x, v)}{\sqrt{2 m(E+e \phi)}} d E$.

Note that, according to our assumption, the electron distribution function depends only on the total energy. Therefore, the function $n_{t}(x, v)$ is an even function of the velocity $v$.

Let us take advantage of the Poisson equation

$\frac{d^{2} \phi(x)}{d x^{2}}=4 \pi \rho$

In terms of the dimensionless variables (10), taking into account that

$\rho(x)=e\left(n_{i}(x)-n_{f}(x)-n_{t}(x)\right)$,

and after simple transformations, it can be rewritten in the form

$\frac{d^{2} \Psi}{d \xi^{2}}=-1+\beta(\Psi)+\alpha(\Psi)$

where the following notations are introduced:

$\alpha(\Psi)=\sqrt{2} \int_{-\Psi}^{0} \frac{n_{t}(v) v_{T} / n_{0}}{\sqrt{E+\Psi}} d E$,

$\beta(\Psi)=n_{f}(\Psi) / n_{0}$.

Note that, at this stage, the form of the potential dependence on the coordinate and cdistribution function of the trapped component remain uncertain. In 

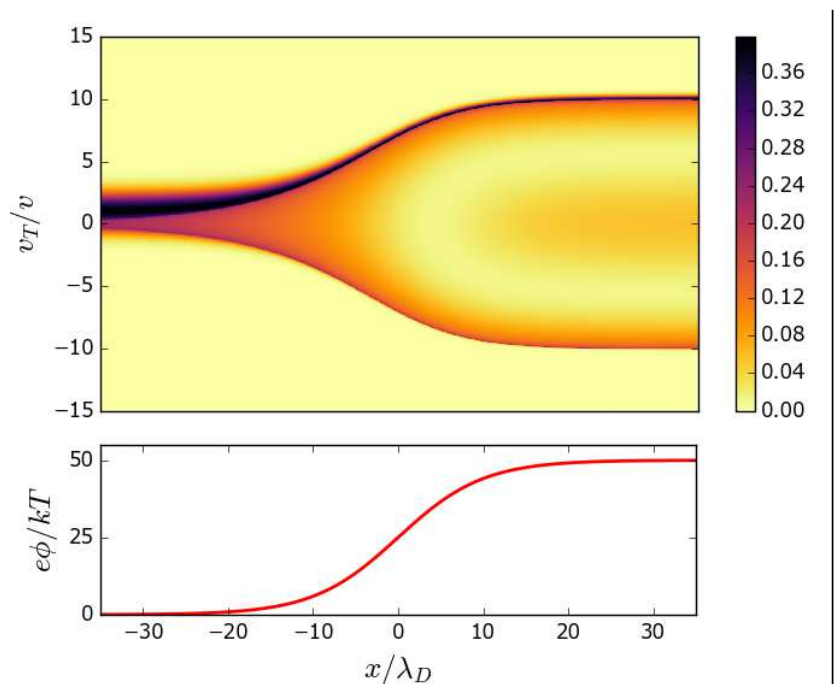

Fig. 2. Phase portrait of the double layer. The bottom panel illustrates the potential profile of the double layer

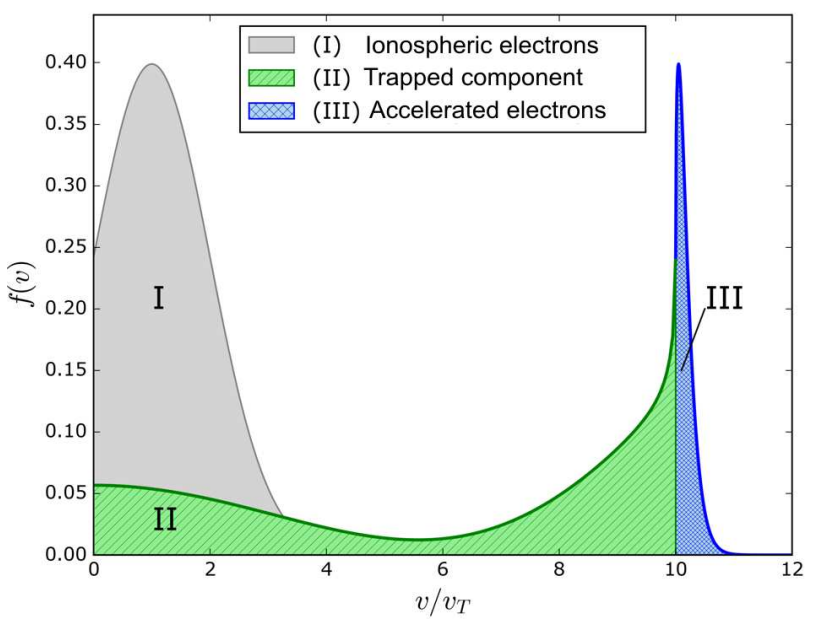

Fig. 3. Limiting distributions of the free and trapped plasma components in the regions before and behind the shock wave

particular, in work [14], it was shown that, with certain restrictions, $n_{t}$ can be taken arbitrary. Depending on the choice of $n_{t}$, Eq. (13) has different solutions for the potential $\Psi(x)$. The specific potential profile depends on the double layer evolution and cannot be unambiguously determined in the framework of the stationary model.

Assuming that $n_{t}$ is a physically feasible distribution of the trapped component, and $\Psi(\xi)$ is a monotonic solution of Eq. (13), which satisfies the conditions $\Psi(-\infty)=0$ and $\Psi(+\infty)=\Psi_{0}$, it is easy to find the distribution $n_{t}$ itself by considering definition
(14) as an integral equation for $n_{t}$. As was marked in work [22], Eq. (14) is an Abel integral equation. Its solution is

$n_{t}(v)=\frac{n_{0} \sqrt{2}}{\pi v_{T}} \int_{0}^{|E|} \frac{\alpha^{\prime}(\theta) d \theta}{\sqrt{-E-\theta}}$.

Below, we will use a model potential $\Psi(\xi)$, which will allow us to determine the distribution function of the trapped component. Let the potential be given in the form

$\Psi(\xi)=\frac{\Psi_{0}}{1+e^{-\xi / \delta}}$,

where $\delta$ is a free parameter that determines the double layer width. For general reasons, the expected value of $\delta$ can amount to a few Debye radii.

Let us determine the quantity $\alpha^{\prime}(\Psi)$. Having expressed the second derivative $d^{2} \Psi(\xi) / d \xi^{2}$ in terms of the potential $\Psi$ itself, we obtain

$\frac{d^{2} \Psi}{d \xi^{2}}=\frac{\Psi_{0}}{\delta^{2}} \Phi(1-2 \Phi)(1-\Phi)$

By differentiating Eq. (13) with respect to $\Psi$, we have

$\alpha^{\prime}(\Psi)=-\beta^{\prime}(\Psi)+\frac{1}{\delta^{2}}\left(6 \Phi^{2}-6 \Phi+1\right)$,

where $\Phi=\Psi / \Psi_{0}$, and the prime means the derivative with respect to $\Psi$. The quantity $\beta^{\prime}(\Psi)$ can be easily found, by using definition (15). Namely,

$\beta^{\prime}(\Psi)=-\sum_{\sigma= \pm 1} \int_{0}^{\infty} \frac{x\left(s-\sigma u_{d}\right)}{2 s \sqrt{\pi \Psi}} \exp \left(-\frac{\left(s-\sigma u_{d}\right)^{2}}{2}\right)$,

where $s=\sqrt{x^{2}+x \sqrt{8 \Psi}}$.

The required integration in formula (16) can be easily performed numerically. The phase portrait of the double layer is depicted in Fig. 2, and the limiting distributions of the free and trapped components at large distances from the double layer are exhibited in Fig. 3.

Let us analyze the results obtained. In Fig. 3, region I (gray) demonstrates the initial distribution in the free component before the acceleration. This component can be identified with the magnetospheric plasma.

Region II (green) illustrates the distribution in the trapped component at $x=\infty$. Note that this

ISSN 2071-0194. Ukr. J. Phys. 2018. Vol. 63, No. 8 
distribution is symmetric and defined in the interval $\left[-v_{\phi},+v_{\phi}\right]$. Two specific features of this component can be distinguished. The maximum located at the center is naturally interpreted as a certain background atmosphere in the wake wave. The maximum located near the characteristic limiting velocity value $-v_{\phi}$ can be considered as a downward electron beam formed by the Io accelerating potential. After being reflected from the double layer, it forms a symmetric maximum near the velocity value $+v_{\phi}$. Note that the specific form of the beam distribution function is a consequence of the artificial choice of the model double-layer potential profile (17). In more realistic models, the distribution function for the trapped component can be different, and the potential profile will differ from expression (17). Nevertheless, the simple model (17) allows the main features of the analyzed phenomenon to be analyzed qualitatively.

Finally, region III (blue) exhibits the free component distribution after the shock wave has passed. These are electrons of the magnetospheric plasma that were accelerated by the double layer potential along the flux tube toward Io and backward from Jupiter. This component generates an upward beam with a low effective temperature, which satisfies the criterion of a transition into the CSR mode (2).

\section{Conclusion}

To summarize, the processes have been considered that can provide conditions for a polarization phase transition into the cyclotron superradiance mode in the region of the Io flux tube foot. A high power of CSR allows the latter to be considered as the main generation mechanism of decameter radio storms of the S-type, with the Jupiter-Io system being their source. It is shown that the plasma double layer in the region of the Io flux tube foot is responsible for the formation of dense cold electron bunches moving upward, for which a phase transition into the CSR mode is possible. The maximum of the effect is observed in the case where the double layer motion velocity is comparable with the thermal velocity of the electron plasma component. This velocity is typical of electrostatic shock waves in plasma. In turn, the shock waves with a double layer can arise as a result of the interaction between the Jovian ionospheric plasma and the electron fluxes accelerated by the Io potential and moving along the Io flux tube.
The work was partially sponsored in the framework of the Program of Fundamental Research of the Department of Physics and Astronomy of the National Academy of Sciences of Ukraine (project No. 0117U000240).

1. T.D. Carr, M.D. Desch, J.K. Alexander. Phenomenology of magnetospheric radio emissions. In Physics of the Jovian Magnetosphere, edited by A.J. Dessler (Cambridge Univ. Press, 1983), p. 226.

2. B.P. Ryabov, N.N. Gerasimova. Sporadic Radio Emission of Jupiter at Decameter Wavelengths (Naukova Dumka, 1990) (in Russian).

3. N. Krupp et al. Dynamics of the Jovian magnetosphere. In Jupiter: Planet, Satellites, Magnetosphere, edited by F. Bagenal et al. (Cambridge Univ. Press, 2004), p. 617.

4. J.T. Clarke et al. Ultraviolet emissions from the magnetic footprints of Io, Ganymede and Europa on Jupiter. Nature 415, 997 (2002).

5. J.E.P. Connerney et al. Jupiter's magnetosphere and aurorae observed by the Juno spacecraft during its first polar orbits. Science 356, 826 (2017).

6. A.P. Fomina. On the quantum nature of quasi-periodic Sbursts of the decameter emission of Jupiter-Io. Dopov. Nat. Akad. Nauk Ukr. 1, 97 (2001).

7. P.I. Fomin, A.P. Fomina. Dicke superradiance on Landau levels. Probl. At. Sci. Technol. 6, 45 (2001).

8. P.I. Fomin, A.P. Fomina, V.N. Mal'nev. The superradiation of magnetized electrons and the power of decameter radiation of the Jupiter-Io system. Ukr. J. Phys. 49, 3 (2004).

9. V.M. Mal'nev, A.P. Fomina, P.I. Fomin. Polarization phase transition to the superradiance regime of the inverted system of electrons on high Landau levels. Ukr. J. Phys. 47, 1001 (2002).

10. O.P. Novak, A.P. Fomina, R.I. Kholodov. Account of the longitudinal temperature in cyclotron superradiance. Probl. At. Sci. Technol. 3, 69 (2013).

11. R.H. Dicke. Coherence in spontaneous radiation processes. Phys. Rev. 93, 99 (1954).

12. V.V. Parkhomchuk, A.N. Skrinskii. Electron cooling: 35 years of development. Usp. Fiz. Nauk 170, 475 (2000) (in Russian).

13. N.S. Dikanskii, V.I. Kudelainen, V.A. Lebedev et al. Limiting possibilities of electron cooling. Preprint IYaF 88-61 (1988).

14. D. Montgomery, G. Joyce. Shock-like solutions of the electrostatic Vlasov equation. J. Plasma Phys. 3, 1 (1969).

15. M.A. Raadu. The physics of double layers and their role in astrophysics. Phys. Rep. 178, 25 (1989).

16. N. Singh, R.W. Schunk. Plasma response to the injection of an electron beam. Plasma Phys. Contr. Fus. 26, 859 (1984).

17. V.I. Maslov. Electron beam reflection from the plasma due to double layer formation. In Proceedings of the 4 th International Workshop on Nonlinear and Turbulent Pro- 
cesses in Physics, edited by V.G. Bar'yakhtar, V.M. Chernousenko, N.S. Erokhin et al. (World Scientific, 1990), p. 898.

18. V.I. Maslov. Double layer formed by relativistic electron beam. Plasma Phys. Fus. Technol. 13, 676 (1992).

19. V.I. Maslov, V.N. Oraevsky, Yu.Ya. Ruzhin. Ion acceleration in collective fields at electron beam injection from spacecraft. Phys. Scr. 57, 453 (1998).

20. V.I. Maslov, I.P. Levchuk, S. Nikonova, I.N. Onishchenko. Occurrence of accelerating field, formation and dynamics of relativistic electron beam near Jupiter. East Eur. J. Phys. 5, 78 (2018).

21. V.I. Maslov, A.P. Fomina, R.I. Kholodov, I.P. Levchuk, S.A. Nikonova, O.P. Novak, I.N. Onishchenko. Accelerating field excitation, occurrence and evolution of electron beam near Jupiter. Probl. At. Sci. Technol. 4, 106 (2018).

22. I.B. Bernstein, J.M. Greene, M.D. Kruskal. Exact nonlinear plasma oscillations. Phys. Rev. 108, 546 (1957).

Received 10.07.18. Translated from Ukrainian by O.I. Voitenko
О. Новак, Р. Холодов, А. Фоміна

РОЛЬ ПОДВІЙНИХ ШАРІВ У ФОРМУВАННІ УМОВ ПОЛЯРИЗАЦЙНОГО ФАЗОВОГО ПЕРЕХОДУ ДО СТАНУ НАДВИПРОМІНЮВАННЯ В СТРУМОВІЙ ТРУБЦІ ІО

$\mathrm{P}$ е $з$ ю м е

Розглянуто можливість фазового переходу електронів магнітосфери Юпітера в режим циклотронного надвипромінювання поблизу основи струмової трубки Іо. Висока потужність циклотронного надвипромінювання дозволяє розглядати його як основний механізм генерації декаметрового випромінювання Юпітера у вигляді S-сплесків. Показано, що низхідні потоки електронів від Іо здатні утворювати подвійні електростатичні шари у вигляді ударних хвиль. Такі хвилі при проходженні вздовж струмової трубки прискорюють електрони магнітосфери, внаслідок чого ефективна температура електронної компоненти значно знижується. В утворених таким чином висхідних потоках наявні сприятливі умови для фазового переходу в режим циклотронного надвипромінювання. 